\title{
EPIPELIC DIATOM COMMUNITIES IN THE ŠERIĆKA BARA MARSH (NORTHEAST BOSNIA AND HERZEGOVINA)
}

\section{Jasmina Kamberović ${ }^{*}$, Koraljka Kralj Borojević ${ }^{2}$,Aldijana Razić ${ }^{3}$ \& Senka Barudanović ${ }^{4}$}

${ }^{1}$ University of Tuzla, Faculty of Natural Sciences and Mathematics,

Univerzitetska 4, BiH-75 000 Tuzla, Bosnia and Herzegovina (e-mail: jasmina.kamberovic@untz.ba)

${ }^{2}$ Warrington, United Kingdom (e-mail: kora.kralj@gmail.com)

${ }^{3}$ University Clinical Center Tuzla, Trnovac bb, BiH-75 000 Tuzla, Bosnia and Herzegovina

(e-mail: aldijanamedic@windowslive.com)

${ }^{4}$ University of Sarajevo, Faculty of Science, Ulica Zmaja od Bosne, BiH-71 000 Sarajevo,

Bosnia and Herzegovina (e-mail: sebarudanovic@gmail.com)

Kamberović, J., Kralj Borojević, K., Razić, A. \& Barudanović, S.: Epipelic diatom communities in the Šerićka Bara marsh (northeast Bosnia and Herzegovina). Nat. Croat., Vol. 26, No. 1., 17-32, Zagreb, 2017.

Epipelic diatom communities were studied in the Šerićka bara marsh in northeast Bosnia and Herzegovina throughout 2009. During spring and summer a total of 57 species was found at 6 sampling points. The highest number of species was noted for genera Gomphonema (8) and Nitzschia (5). The most abundant species were Melosira varians C.Agardh, Gyrosigma acuminatum (Kützing) Rabenhorst, Lemnicola hungarica (Grunow) F.E.Round \& P.W.Basson, Navicula cryptocephala Kützing, Cocconeis placentula var. placentula Ehrenberg, Hippodonta capitata (Ehrenberg) Lange-Bertalot, Metzeltin \& Witkowski, Fallacia pygmaea (Kützing) A.J.Stickle \& D.G.Mann, Anomoeoneis sphaerophora E.Pfitzer, Craticula cuspidata (Kutzing) D.G.Mann, Cyclotella meneghiniana (Kutzing) D.G.Mann and Tryblionella hungarica (Grunow) Frenguelli. The spatial variation of the epipelic community was higher in summer as summarized by PCO analysis. Values of the Shannon-Wiener Diversity Index ranged from 2.47 to 2.96, while the Rott Trophic Index ranged from 2.7 to 3.4. Most of the species were eutraphentic (61\%) and alkaliphilous $(60 \%)$, indicating the eutrophic and polytrophic status of this wetland.

Key words: epipelon, diatoms, biodiversity, eutrophication, ecological status, wetland, Bosnia and Herzegovina, Šerićka bara

Kamberović, J., Kralj Borojević, K., Razić, A. \& Barudanović, S.: Epipelonske dijatomeje u Šerićkoj bari (sjeveroistočna Bosna i Hercegovina). Nat. Croat., Vol. 26, No. 1., 17-32, Zagreb, 2017.

U radu su istraživane epipelonske zajednice dijatomeja tijekom 2009. godine u Šerićkoj bari u sjeveroistočnoj Bosni i Hercegovini. Determinirano je 57 vrsta na 6 lokaliteta u proljetnoj i ljetnoj sezoni uzorkovanja. Rodovi najbrojniji vrstama su Gomphonema (8) i Nitzschia (5), a dominantne vrste bile su Melosira varians C.Agardh, Gyrosigma acuminatum (Kützing) Rabenhorst, Lemnicola hungarica (Grunow) F.E.Round \& P.W.Basson, Navicula cryptocephala Kützing, Cocconeis placentula var. placentula Ehrenberg, Hippodonta capitata (Ehrenberg) Lange-Bertalot, Metzeltin \& Witkowski, Fallacia pygmaea (Kützing) A.J.Stickle \& D.G.Mann, Anomoeoneis sphaerophora E.Pfitzer, Craticula cuspidata (Kutzing) D.G.Mann, Cyclotella meneghiniana Kutzing i Tryblionella hungarica (Grunow) Frenguelli. Struktura epipelonske zajednice bila je prostorno raznolikija u ljetnoj u odnosu na proljetnu sezonu uzorkovanja. ShannonWienerov indeks raznolikosti iznosio je od 2,47 do 2,96, dok se trofički indeks prema Rott-u kretao $\mathrm{u}$ rasponu od 2,7 do 3,4. Najveći broj vrsta pripadao je kategoriji alkalofilnih (60\%) i eutrofnih (61\%) pokazatelja i upućuje na eutrofni i politrofni status močvare.

Ključne riječi: epipelon, dijatomeje, bioraznolikost, eutrofikacija, ekološki status, Bosna i Hercegovina, Šerićka bara. 


\section{INTRODUCTION}

Epipelon is a community of autotrophic microorganisms (cyanobacteria, algae) on the sediment at the bottom of stagnant and running waters (PoulíčKoví et al., 2008). The first studies on algal epipelic communities were performed by Round (1953). Epipelic communities are significant primary producers in shallow marshes (Velasco et al., 2003; ŠPAČKOvÁ et al., 2009), thus being important in water quality assessment (Gómez, 1998), trophic interactions (Moulten et al., 2004) and regulation of the nutrient cycle between water and sediment (DoDs, 2003). Algal communities reflect environmental conditions and their community structure indicates the trophic status of the ecosystem (YANG \& DicKMAN, 1993).

Compared to that of phytoplankton and epiphyton, the importance of the epipelic communities of lake ecosystems is often greatly underestimated, thus needing a comprehensive review (PoulíčKová et al., 2008; 2014).

Wetlands are among the most endangered ecosystems in Bosnia and Herzegovina and are, due to permanent land reclamation and drainage, limited to small areas. The First National Report of Bosnia and Herzegovina for the Biodiversity Convention recognized wetland areas around Lake Modrac and Šerićka bara marsh as important refuge habitats (ReDžić et al., 2008). During the last several decades Šerićka bara has been used as a hunting ground, and thus lacks any form of legal protection. Currently, there is an ongoing local legislative procedure in progress (www.vladatk.kim.ba, 2017), with a proposal to establish a fifth category of protected area according to IUCN categorization. The only published data about the biological diversity of the Šerićka bara marsh comprised birds and insects (KuLIJER \& ŠIMIć, 2011). There are no records of previous algological and floristic research into this wetland.

In general, diatom research into the wetlands in Bosnia and Herzegovina is extremely scarce. HAFNER et al. (2013) investigated epiphytic diatoms of the sub-Mediterranean wetland Hutovo blato, whilst phytoplankton communities in Bardača (northern Bosnia) were studied by LoLIć et al. (2015).

This study of epipelic diatom communities presents the first insight into the algal assemblages of the Serićka bara marsh. The objectives of the study were to: (i) describe the diatom epipelic communities of the Šerićka bara marsh, (ii) compare the community structure in spring and summer, and (iii) assess the trophic status of the wetland.

\section{STUDY AREA}

Šerićka bara marsh is situated in northeastern Bosnia and Herzegovina at the south side of the mouth of Spreča River into Lake Modrac. It originated after the formation of Lake Modrac, by the deposition of sediment from Spreča River and the formation of the bank that has separated it from the lake for the last 30 years. This permanent wetland ecosystem is fed by several small surface streams flowing from the surrounding settlements. It is overgrown by thick vegetation of reeds and rushes, submerged and floating aquatic plants. The climate is moderate continental. Physical properties of the study area are presented in Tab. 1 (obtained using Google Earth 2010/Tele Atlas 2010/Image 2010 Digital Globe and field research).

Algal samples were collected at 6 sampling points during two seasons (Fig. 1). Most sampling points (L2-L6) were shaded by floating vegetation of Lemnaceae species, especially in the summer. 
Tab. 1. Physical properties of the study site

\begin{tabular}{|c|c|c|c|c|c|c|}
\hline \multicolumn{2}{|c|}{ Geographic position } & $\begin{array}{l}\text { Altitude } \\
\text { (m a.s.l.) }\end{array}$ & \multirow{2}{*}{$\begin{array}{c}\text { Max. length } \\
(\mathrm{m})\end{array}$} & \multirow{2}{*}{$\begin{array}{l}\text { Max. width } \\
(\mathrm{m}) \\
250\end{array}$} & \multirow{2}{*}{$\begin{array}{c}\text { Max. depth } \\
(\mathrm{m})\end{array}$} & \multirow{2}{*}{$\begin{array}{c}\begin{array}{c}\text { Surface } \\
\text { (ha) }\end{array} \\
30\end{array}$} \\
\hline $44^{\circ} 28^{\prime} 17^{\prime \prime}$ & $18^{\circ} 34^{\prime} 02^{\prime \prime}$ & 198 & & & & \\
\hline
\end{tabular}
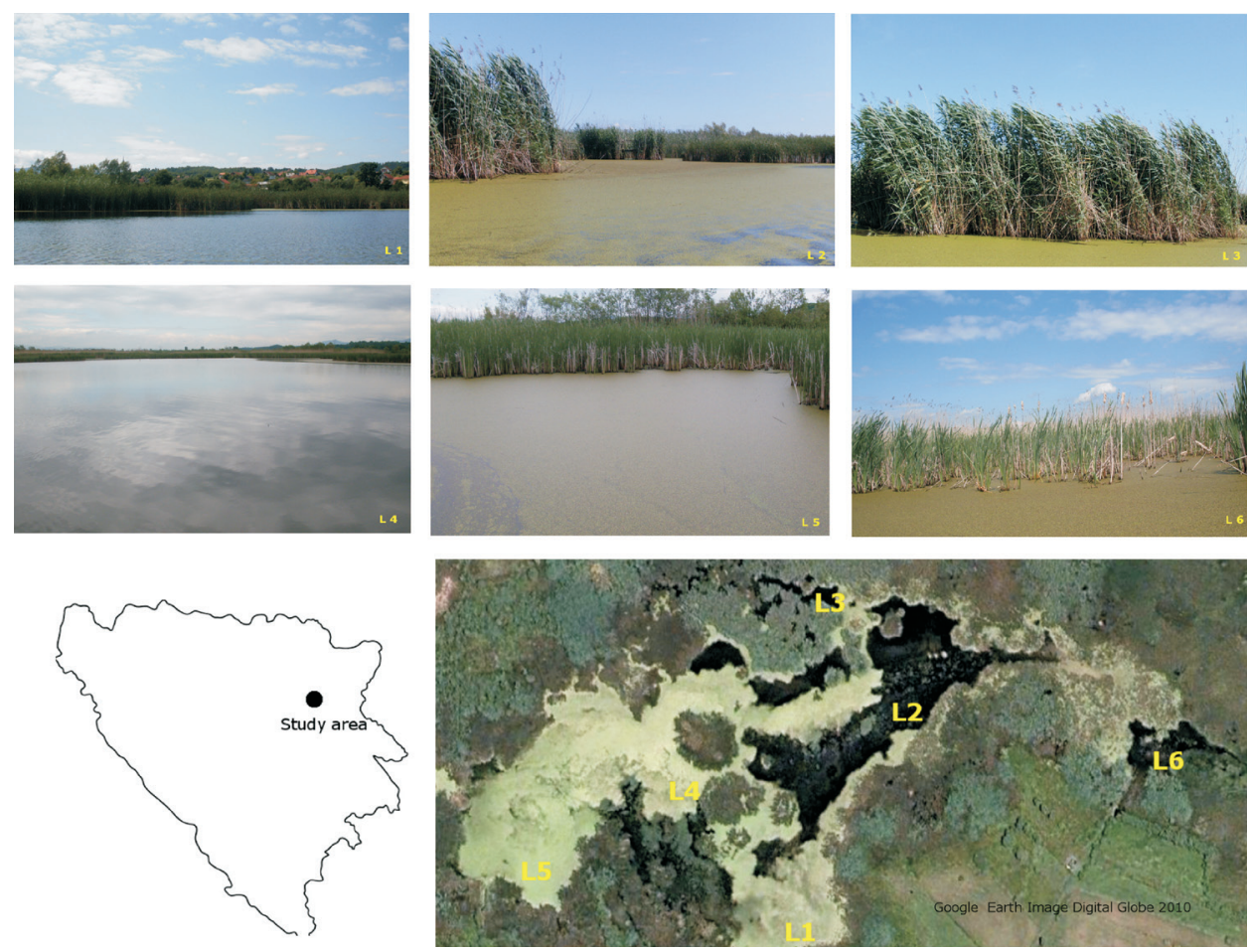

Fig. 1. Study area and sampling localities of the Šerićka bara marsh

\section{MATERIALS AND METHODS}

Temperature and $\mathrm{pH}$ were measured using a standard thermometer and a WT $-\mathrm{pH}$ inolab probe (Xylem Analytics Germany GmbH \& Co. KG.). Transparency was measured with Secchi disk. Organic matter was determined using the oxidation method with potassium permanganate (TrajKović et al., 1983)

Diatom samples were collected two times, by pipetting mud at 6 sampling points in spring and summer of 2009. Samples were preserved with $4 \%$ formaldehyde. Diatoms were acid cleaned following Hustedt (1930) and identified on permanent slides prepared using Naphrax (Brunel Microscopes Ltd., U.K.). Algae were identified using light microscopes BA310 Motic optical microscope (Speed Fair Co. Ltd., China) and Olympus BX41 (Olympus Corporation, Japan) at a magnification of 1000x and following Krammer \& LAnge-Bertalot (1986; 1988; 1991a; 1991b), Krammer $(2001 ; 2000 ; 2002)$ and HofmanN et al. (2011). Microphotographs were taken with Optica Pro 3LT camera and processed with Optika Vision Pro Software (Optika Microscopes, Italy). Relative abundance was 
calculated by counting up to 400 frustules on permanent slides. Nomenclature was double checked following the Algaebase webpage (GUIRY \& GUIRY, 2015). Trophic status was determined using Trophic Index according to Rотт et al. (1999), representing the average of indicator values weighted by relative abundance, and using the Trophic Index according to VAN DAM et al. (1994), representing the arithmetic average of species indicator values. Biodiversity of species was estimated for each sample using the ShannonWiener diversity index (SHANNON \& Wiener, 1949).

All analyses were performed using the software PRIMER-E (CLARKE \& GORLEY, 2006) with the add-on package PERMANOVA+ (ANDERson et al., 2008). PERMANOVA analysis (4999 permutations) based on Bray-Curtis similarity matrix was used to test for statistical differences on the species composition between two sampled periods. Principal coordinates analysis (PCO) was used for the visualisation of the multivariate patterns of the epipelic composition (ANDERson et al., 2008).

\section{RESULTS}

The average water temperature in the spring sampling period was $18^{\circ} \mathrm{C}$, while higher values were noted in summer, reaching $22.3^{\circ} \mathrm{C}$. Transparency was lower in spring than in summer, $24 \mathrm{~cm}$ and $31.3 \mathrm{~cm}$ respectively. Water colour was mostly brown, indicating a probable influence by soil substances. The consumption of potassium permanganate $\left(\mathrm{KMnO}_{4}\right)$ ranged from 20.3 to $44.3 \mathrm{mg} \mathrm{L}^{-1}$, indicating a greater presence of organic material in the water. Water was slightly alkaline with $\mathrm{pH}$ values ranging from 6.8 to 8 (Tab. $2)$. Statistically significant $(p<0.05)$ positive correlations between temperature and permanganate value $(\mathrm{r}=0.6)$ and negative correlation between temperature and $\mathrm{pH}(\mathrm{r}-6.9)$ were established using Pearson's correlation coefficient.

A total of 57 diatom species belonging to 33 genera were noted in epipelic samples of the Šerićka bara marsh. Most diatom species belonged to the genera Gomphonema (8) and Nitzschia (5). The number of species in a sample ranged from 18 to 32. The most abundant species were Melosira varians C.Agardh, Gyrosigma acuminatum (Kützing) Rabenhorst, Lemnicola hungarica (Grunow) F.E.Round \& P.W.Basson, Navicula cryptocephala Kützing, Cocconeis placentula var. placentula (Ehrenberg) Grunow, Hippodonta capitata (Ehrenberg) Lange-Bertalot, Metzeltin \& Witkowski, Fallacia pygmaea (Kützing) A.J.Stickle \& D.G.Mann, Anomoeoneis sphaerophora E.Pfitzer, Craticula cuspidata (Kutzing) D.G.Mann, Cyclotella meneghiniana Kützing and Tryblionella hungarica (Grunow) Frenguelli (Fig. 2). Shannon-Wiener index ranged from 2.47 to 2.96, indicating high diversity in the epipe-

Tab. 2. Environmental characteristics of sampling points (first values represent spring, and second summer measurements)

\begin{tabular}{|l|c|c|c|c|c|c|}
\hline Sampling point & L1 & L2 & L3 & L4 & L5 & L6 \\
\hline Water temperature $\left({ }^{\circ} \mathrm{C}\right)$ & $18-22$ & $18-22$ & $17,5-22$ & $18-24$ & $19-22$ & $18-22$ \\
\hline $\mathrm{pH}$ & $7.5-7.1$ & $7.5-6.8$ & $7.4-7$ & $7.5-7$ & $8-7.4$ & $7.5-7.3$ \\
\hline Transparency $(\mathrm{cm})$ & $15-25$ & $16-27$ & $25-23$ & $17-28$ & $16-32$ & $55-53$ \\
\hline Depth $(\mathrm{cm})$ & $45-45$ & $66-51$ & $40-36$ & $47-40$ & $45-40$ & $55-53$ \\
\hline Permanganate value $\left(\mathrm{mg} \mathrm{L}^{-1} \mathrm{KMnO}_{4}\right)$ & $25.3-35.5$ & $34.2-39.3$ & $20.3-50.7$ & $29.8-44.3$ & $30.4-30.4$ & $32.9-25.3$ \\
\hline Coverage by floating vegetation $(\%)$ & $0-30$ & $5-95$ & $20-100$ & $0-100$ & $100-100$ & $100-35$ \\
\hline
\end{tabular}




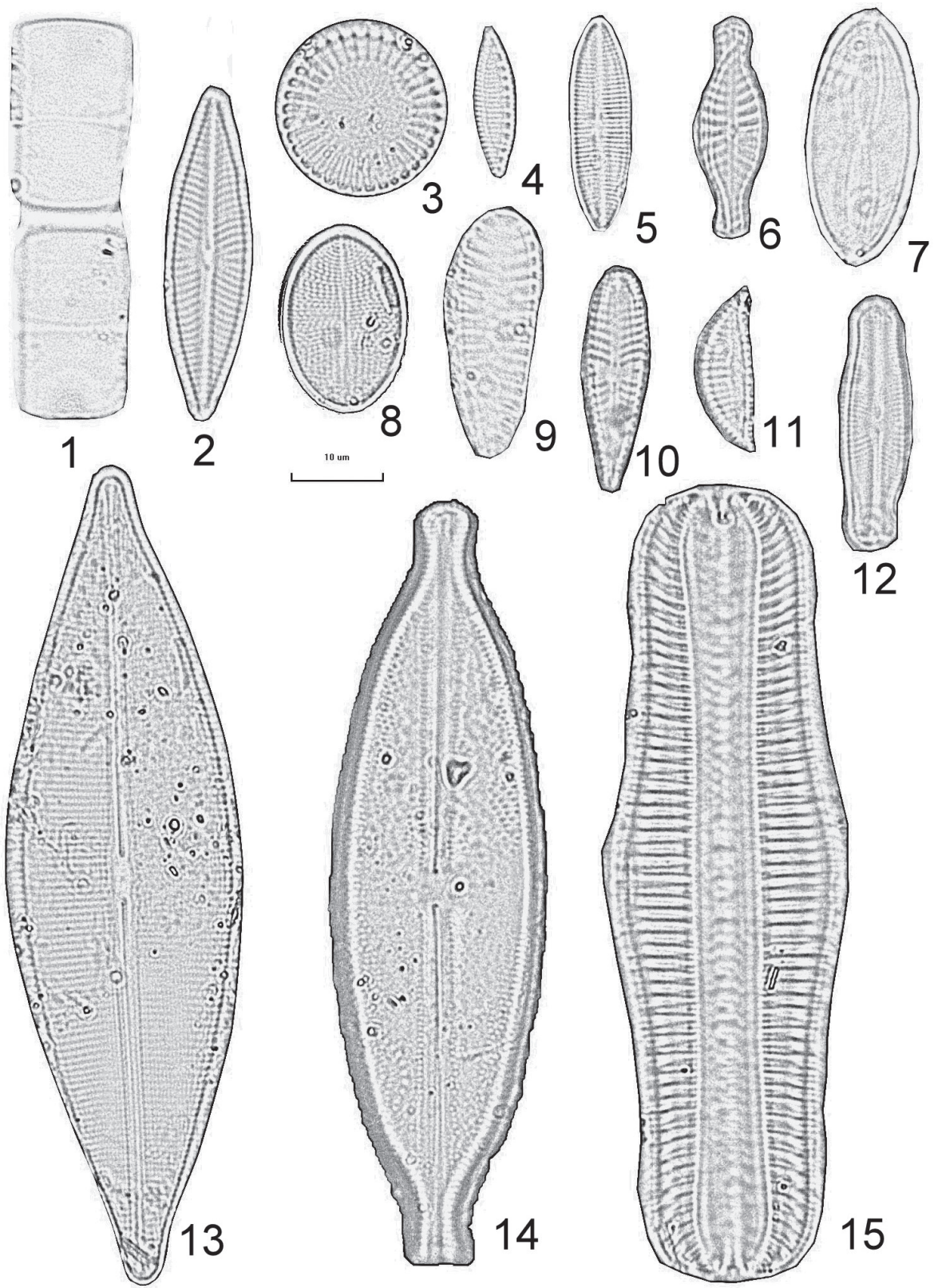

Fig. 2. The most abundant diatoms in the Šerićka bara marsh

1 -Melosira varians C.Agardh, 2 - Navicula cryptocephala Kützing, 3-Cyclotella meneghiniana Kützing, 4-Nitzschia frustulum (Kützing) Grunow, 5 - Lemnicola hungarica (Grunow) F.E.Round \& P.W.Basson, 6 - Hippodonta capitata (Ehrenberg) Lange-Bertalot, Metzeltin \& Witkowski, 7 - Fallacia pygmaea (Kützing) A.J.Stickle \& D.G.Mann, 8 - Cocconeis placentula var. placentula Ehrenberg, 9 - Surirella terricola Lange-Bertalot \& E.Alles, 10 - Gomphonema olivaceum (Hornemann) Brébisson, 11 - Encyonema minutum (Hilse) D.G.Mann, 12 - Sellaphora pupula sensu lato, 13 - Craticula cuspidata (Kutzing) D.G.Mann, 14 - Anomoeoneis sphaerophora E.Pfitzer, 15 - Rhopalodia gibba (Ehrenberg) Otto Müller 

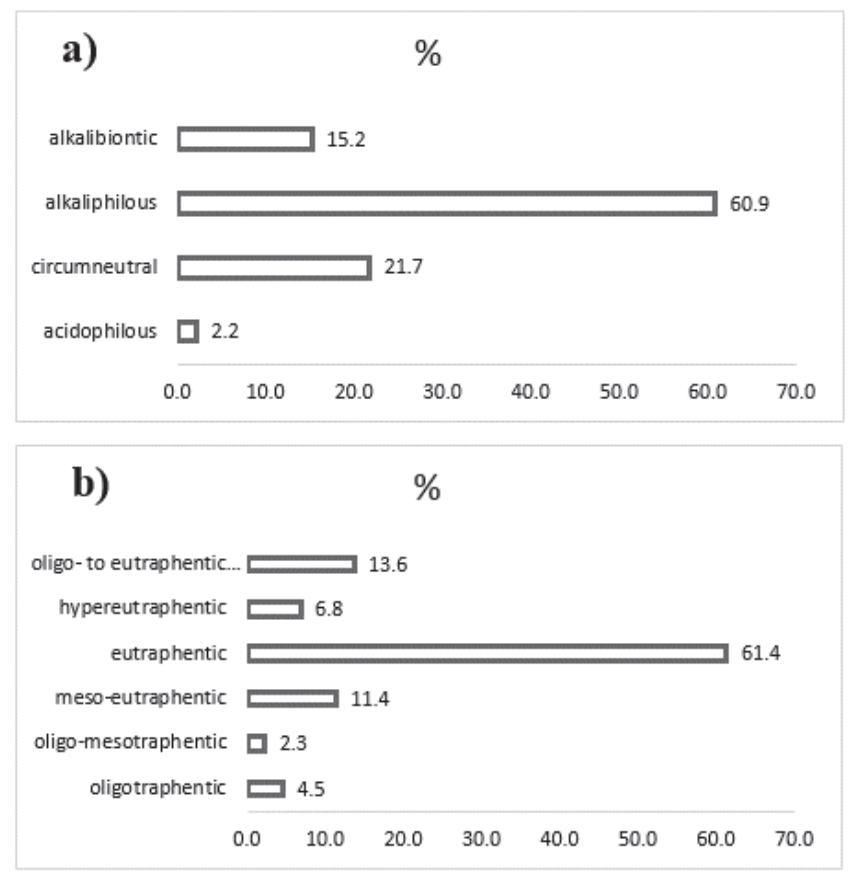

Fig. 3. Representation of percentages of diatoms in relation to (a) indicator values of water reaction and (b) trophic status by vAN DAM et al., 1994.

lic communities. Rott Trophic Index varied between 2.7 and 3.4, thus indicating a eupolytrophic to polytrophic status for the marsh (Tab. 3). Indicator values for water reaction (R) and trophic status (T) by VAN DAM et al. (1994) were established for the majority of identified species ( $80 \%$ and $77 \%$, respectively). Most of the identified species (Fig. 3) were eutraphentic (61\%) and alkaliphilous (60\%). The average indicator trophic value (van DAm et al., 1994) for all identified species in all samples was 4.97, which corresponds to a eutraphentic status for the wetland.

The epipelic community showed higher spatial variation in the summer, as summarized by PCO analysis (Tab. 4). The first two axes accounted for approximately $55.8 \%$ of the total variance. The first PCO axis was best described by water transparency $(\mathrm{r}-0.58)$ and coverage by floating vegetation $(\mathrm{r}-0.46)$, while the second axis was described by water temperature $(r=0.59)$ and transparency $(r=0.71)$. Summer samples were mostly distributed along the PCO Axis 1, mainly differing in the percentage of coverage by floating vegetation. Positive values of Pearson's correlation coefficient with the first PCO axis were noted for the following species: Anomoeoneis sphaerophora, Nitzshia frustulum (Kützing) Grunow and Tryblionella hungarica. Negative correlation coefficient values have been established for the species Cocconeis placentula var. euglypta (Ehrenberg) Grunow, Navicula radiosa Kützing, Navicula cryptocephala and Nitzschia palea (Kützing) W.Smith. Positive values of Pearson's correlation coefficient $(r>0.5)$ with the second PCO axis and higher water temperature were noted for the species Cyclotella meneghiniana, Anomoeoneis sphaerophora, Gomphonema pumilum (Grunow) E.Reichardt \& Lange-Bertalot and Gomphonema psedoaugur Lange-Bertalot. Negative values of Pearson's correlation coeffi- 


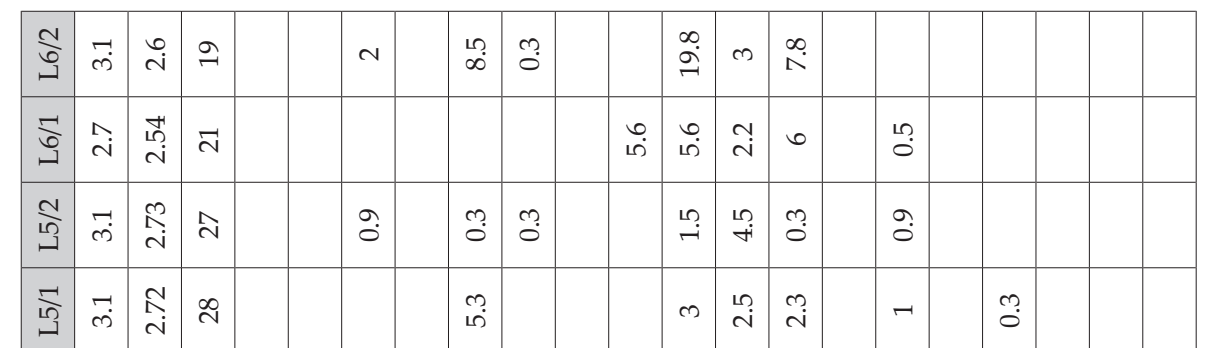

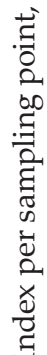

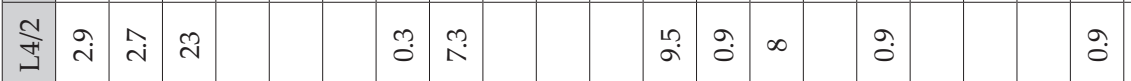

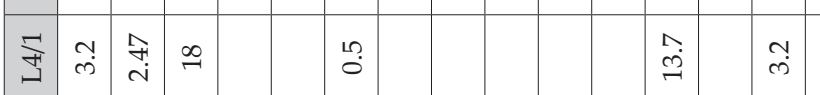

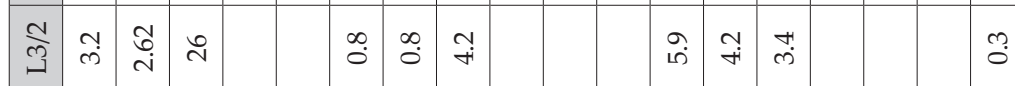

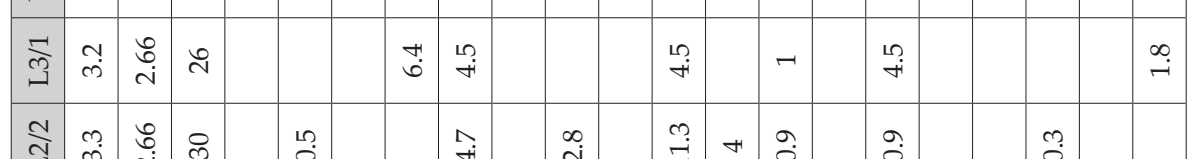

\begin{tabular}{|c|c|c|c|c|c|c|c|c|c|c|c|c|c|c|c|}
\hline 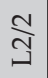 & $\stackrel{m}{\oplus}$ & $\begin{array}{l}\stackrel{0}{0} \\
\stackrel{i}{ }\end{array}$ & ిల & $\stackrel{10}{0}$ & $\stackrel{\nearrow}{\not}$ & $\begin{array}{l}\infty \\
i \\
i\end{array}$ & $\stackrel{m}{g}$ & $H$ & $\hat{\sigma}$ & & $\grave{o}$ & & & $\stackrel{3}{0}$ & \\
\hline ন & $\stackrel{\sim}{n}$ & 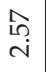 & શิ & $\tilde{o}$ & 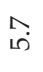 & $\stackrel{L}{O}$ & $\begin{array}{l}0 \\
\dot{0}\end{array}$ & & $\tilde{o}$ & & $\stackrel{g}{0}$ & & & & $\stackrel{\infty}{0}$ \\
\hline$\underset{\exists}{\stackrel{V}{J}}$ & $\stackrel{H}{\oplus}$ & $\widehat{\mathrm{N}}$ & సิ & & $\stackrel{\infty}{\stackrel{\infty}{\sim}}$ & & $\stackrel{\llcorner}{\mathrm{N}}$ & تִ & $\stackrel{\infty}{0}$ & $\dddot{m}$ & $\stackrel{\infty}{0}$ & & $\stackrel{L}{O}$ & & $\stackrel{\infty}{0}$ \\
\hline$\underset{\Xi}{\Xi}$ & $\ddot{\oplus}$ & $\begin{array}{l}\text { ڤ̆ } \\
\text { ì }\end{array}$ & लै & ָָ & $\hat{o}$ & & $\Lambda$ & $\hat{\mathrm{i}}$ & $\stackrel{n}{\rightarrow}$ & & $\hat{o}$ & $\stackrel{3}{0}$ & $\stackrel{\leftrightarrow}{0}$ & & $\stackrel{3}{0}$ \\
\hline
\end{tabular}

\begin{tabular}{|c|c|c|c|c|c|c|c|c|c|c|c|c|c|c|c|c|c|c|c|c|c|}
\hline & & & & 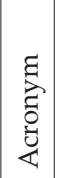 & 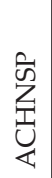 & 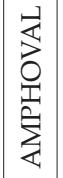 & 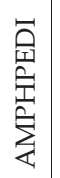 & 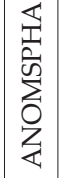 & 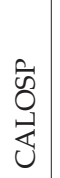 & \begin{tabular}{|l}
$\vec{D}$ \\
$y$ \\
$\bar{y}$ \\
$y$ \\
0 \\
0
\end{tabular} \mid & $\begin{array}{l}\vec{J} \\
\vec{J} \\
\tilde{U} \\
0 \\
\circlearrowright\end{array}$ & $\left|\begin{array}{l}\vec{z} \\
\vec{U} \\
\tilde{U} \\
0 \\
\mathcal{U}\end{array}\right|$ & 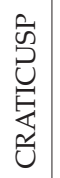 & $\begin{array}{l}\sum_{Z} \\
\sum_{\Perp}^{\prime} \\
己 \\
己 \\
己\end{array}$ & 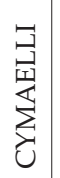 & 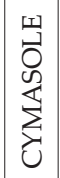 & 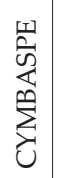 & 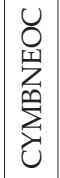 & 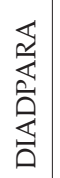 & 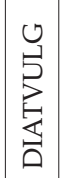 & 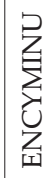 \\
\hline ஸे & 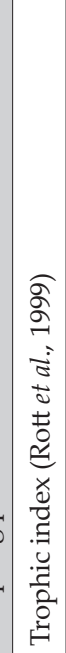 & 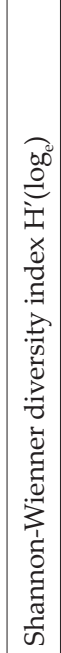 & 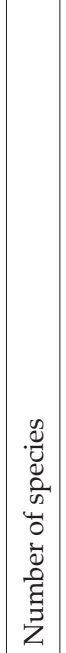 & 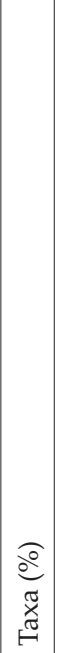 & 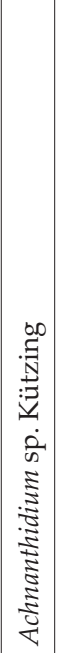 & 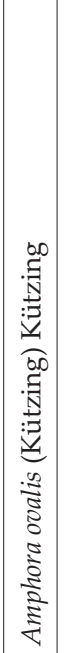 & 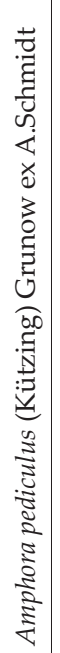 & 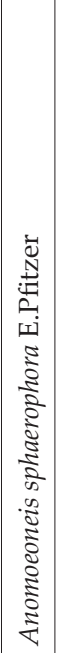 & 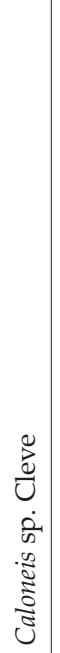 & 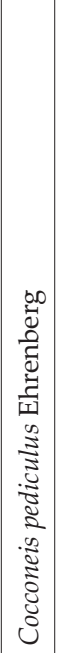 & 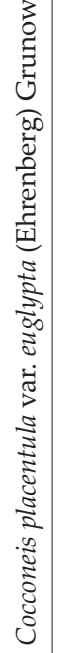 & 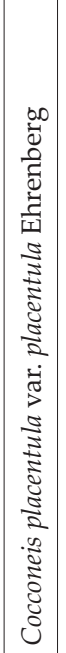 & 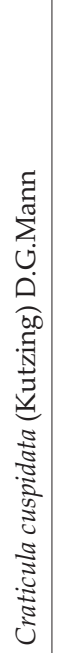 & 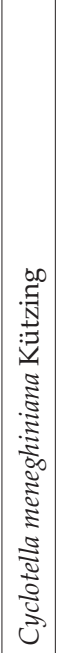 & 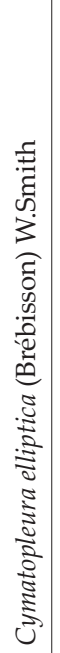 & 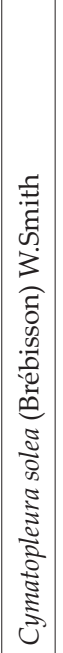 & 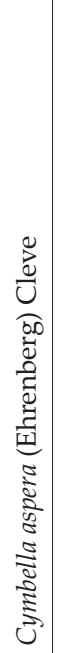 & 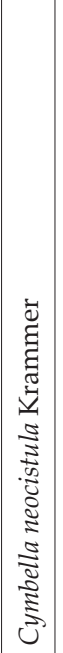 & 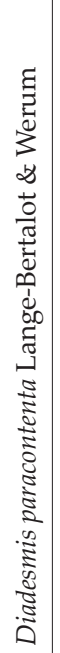 & 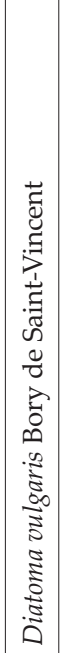 & 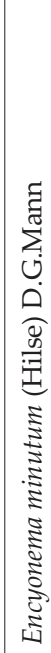 \\
\hline
\end{tabular}

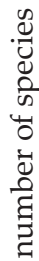




\begin{tabular}{|c|c|c|c|c|c|c|c|c|c|c|c|c|c|c|c|c|c|c|c|c|}
\hline 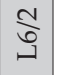 & & $\stackrel{+}{-}$ & & & $\theta$ & & & & & & & & $\stackrel{\llcorner}{+}$ & $\stackrel{\infty}{+}$ & ?ై & $\stackrel{\infty}{-\infty}$ & $\stackrel{m}{m}$ & 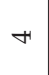 & 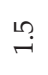 & $\stackrel{\infty}{\rightarrow}$ \\
\hline$\frac{5}{\sigma}$ & & $\stackrel{0}{0}$ & $\ddot{n}$ & & & & & & & & & & & $\stackrel{ت}{ت}$ & $\stackrel{\leftrightarrow}{\sigma}$ & $\stackrel{n}{\rightarrow}$ & $\stackrel{\operatorname{Ln}}{\rightarrow}$ & $\Lambda$ & $\stackrel{0}{\sim}$ & $\begin{array}{l}\infty \\
\stackrel{\infty}{\sim}\end{array}$ \\
\hline$\frac{\sqrt{3}}{\sqrt[n]{3}}$ & $\stackrel{a}{0}$ & & $\grave{o}$ & & $\stackrel{10}{\oplus}$ & & & & & $\grave{o}$ & & $\dot{o}$ & $\stackrel{\operatorname{Ln}}{+}$ & 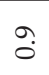 & $\ddot{\sigma}$ & $\stackrel{10}{\circ}$ & $\stackrel{L}{\mathrm{i}}$ & $\begin{array}{l}\infty \\
\stackrel{\infty}{n} \\
\stackrel{n}{2}\end{array}$ & 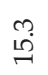 & 0 \\
\hline$\frac{5}{13}$ & $\stackrel{\sim}{\rightarrow}$ & & & & గై & & $\stackrel{\infty}{\infty}$ & $\stackrel{\infty}{\infty}$ & Lִ & & $\stackrel{10}{0}$ & $\stackrel{10}{0}$ & & & $r$ & $\stackrel{3}{\rightarrow}$ & $\infty$ & $\stackrel{\infty}{\underset{j}{v}}$ & $\begin{array}{l}\infty \\
\infty \\
\infty\end{array}$ & \\
\hline 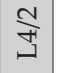 & & & & $\stackrel{\infty}{\stackrel{\infty}{+}}$ & 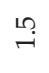 & & & & & & & & $\stackrel{\operatorname{Ln}}{\mathrm{N}}$ & & $\begin{array}{l}\infty \\
\stackrel{\infty}{\infty}\end{array}$ & $\stackrel{L}{\infty}$ & $\exists$ & $\begin{array}{l}\stackrel{3}{ } \\
\stackrel{2}{\sim}\end{array}$ & $\ddot{\sigma}$ & $\overbrace{0}^{0}$ \\
\hline$\stackrel{\leftrightarrows}{\leftrightarrows}$ & & & & $\stackrel{10}{0}$ & $\begin{array}{l}\infty \\
10 \\
10\end{array}$ & $\stackrel{\sim}{N}$ & & กี & ָ̃ & & & & & & 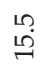 & 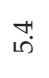 & 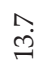 & $\stackrel{\curvearrowright}{\curvearrowright}$ & $\stackrel{\infty}{\infty}$ & \\
\hline$\frac{3}{\frac{3}{n}}$ & & & & & $\underset{\not}{\sim}$ & & & $\stackrel{\infty}{0}$ & & & & $\stackrel{10}{0}$ & $\stackrel{\infty}{0}$ & & 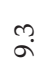 & $\begin{array}{l}\text { Ln } \\
\text { Ln }\end{array}$ & $\stackrel{?}{\stackrel{?}{a}}$ & $\stackrel{\text { }}{\mathrm{d}}$ & $\begin{array}{l}10 \\
10\end{array}$ & $\stackrel{\curvearrowright}{\sim}$ \\
\hline$\frac{\vec{c}}{3}$ & & & $\stackrel{n}{0}$ & & $\stackrel{\infty}{\sim}$ & & $\neg$ & $\stackrel{\infty}{0}$ & & & & $\stackrel{10}{0}$ & $\stackrel{10}{0}$ & $\stackrel{10}{\circ}$ & $\stackrel{M}{+}$ & $\stackrel{-}{\circ}$ & $\stackrel{\text { חִ }}{=}$ & $\stackrel{m}{\stackrel{m}{\longrightarrow}}$ & $\underset{\sim}{\simeq}$ & $\neg$ \\
\hline$\frac{7}{n}$ & & $\stackrel{3}{0}$ & $\stackrel{3}{0}$ & $\stackrel{m}{0}$ & & $\stackrel{10}{0}$ & $\stackrel{\infty}{\circ}$ & $\hat{\sigma}$ & & & $\stackrel{\infty}{0}$ & & $m$ & & $\begin{array}{l}\infty \\
\stackrel{\infty}{n} \\
\end{array}$ & $\stackrel{\text { Lִ }}{\rightarrow}$ & $\stackrel{\mathcal{I}}{\sim}$ & $\infty$ & $a$ & \\
\hline$\vec{\lambda}$ & $\stackrel{1}{0}$ & & & & $H$ & & $\grave{o}$ & $\hat{o}$ & Lִ & & & & 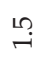 & $\dot{o}$ & 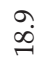 & $\ddot{\infty}$ & $\begin{array}{l}\infty \\
\stackrel{i}{\sim}\end{array}$ & $\stackrel{n}{\vec{N}}$ & $\stackrel{m}{i}$ & \\
\hline$\stackrel{J}{J}$ & $\stackrel{10}{0}$ & & & & $\stackrel{\infty}{\sim}$ & $\overrightarrow{\mathrm{i}}$ & & & $\stackrel{\infty}{0}$ & & & & $\stackrel{\sim}{\infty}$ & & مب & $\stackrel{\infty}{\underset{+}{+}}$ & & + & $\stackrel{m}{m}$ & \\
\hline$\stackrel{\Xi}{\Xi}$ & & & $\stackrel{3}{0}$ & & స̃ & $\hat{\mathrm{i}}$ & $\stackrel{\llcorner}{0}$ & & & & & ָี & $\hat{o}$ & & $\Omega^{3}$ & $\widehat{\grave{i}}$ & $\stackrel{m}{\sim}$ & $\infty$ & $\ddot{\circ}$ & \\
\hline & $\begin{array}{l}\infty \\
0 \\
\frac{\pi}{d} \\
z \\
z \\
z\end{array}$ & $\begin{array}{l}0 \\
3 \\
z \\
z \\
z\end{array}$ & 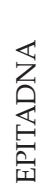 & $\begin{array}{l}\text { w } \\
\bigcirc \\
Z_{1}\end{array}$ & $\begin{array}{l}\sum_{0} \\
\vdots \\
\text { 空 }\end{array}$ & 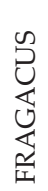 & 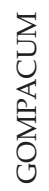 & $\begin{array}{l}\sum_{0} \\
Z \\
\sum \\
\sum \\
0 \\
0\end{array}$ & 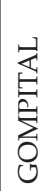 & 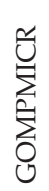 & $\begin{array}{l}z \\
0 \\
0 \\
0 \\
0\end{array}$ & 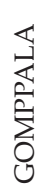 & 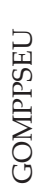 & 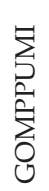 & 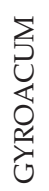 & 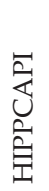 & 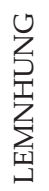 & 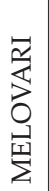 & 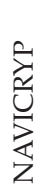 & 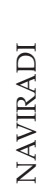 \\
\hline
\end{tabular}
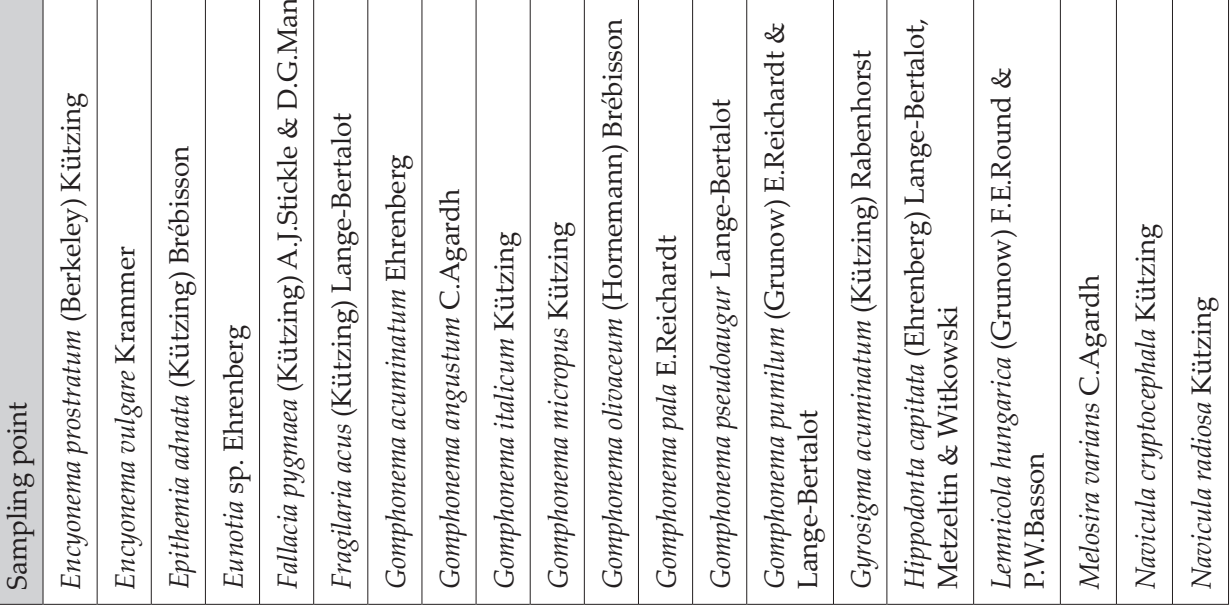


\begin{tabular}{|c|c|c|c|c|c|c|c|c|c|c|c|c|c|c|c|c|c|c|c|c|}
\hline$\frac{-1}{\mathfrak{d}}$ & & & & & & & & & & & & & & & & & $\stackrel{?}{\longrightarrow}$ & $\stackrel{\operatorname{Ln}}{+}$ & & $\stackrel{\infty}{i}$ \\
\hline$\frac{5}{6}$ & & & & $\begin{array}{l}\text { ம) } \\
\text { ம் }\end{array}$ & & $\stackrel{10}{0}$ & & & $ت$ & & 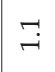 & & 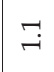 & & & & & N & & $\stackrel{\ln }{+}$ \\
\hline$\frac{1}{13}$ & $\grave{o}$ & $\stackrel{\llcorner}{\infty}$ & $\grave{o}$ & $\stackrel{m}{\text { m) }}$ & & & & $\grave{o}$ & & & & & & & & & & ஸृ & & $\grave{o}$ \\
\hline 5 & & $\stackrel{\text { Pִ }}{\rightarrow}$ & & $N$ & & & & $\stackrel{10}{\circ}$ & & $\dot{\sigma}$ & & $\tilde{\sigma}$ & $\stackrel{\text { กִ }}{\longrightarrow}$ & $\stackrel{\text { ?ִ }}{\longrightarrow}$ & & & $\stackrel{m}{\longrightarrow}$ & $\stackrel{\infty}{+}$ & & $\stackrel{m}{\sim}$ \\
\hline$\underset{J}{\stackrel{J}{J}}$ & $\stackrel{3}{0}$ & & & $\stackrel{m}{+}$ & & & & $\stackrel{10}{0}$ & & $N$ & & $\stackrel{n}{\rightarrow}$ & & & & $\stackrel{n}{-}$ & & & & - \\
\hline$\underset{\exists}{J}$ & & ?ִ & & & & & & & 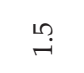 & & & $\stackrel{\sim}{\infty}$ & & & & & & $\stackrel{N}{N}$ & & $\stackrel{n}{\sim}$ \\
\hline$\frac{N}{3}$ & & in & & $\stackrel{\text { Lִ }}{O}$ & & & & & $\stackrel{10}{\circ}$ & & $\stackrel{\curvearrowright}{\rightarrow}$ & $\stackrel{\curvearrowright}{\curvearrowright}$ & & $\stackrel{3}{0}$ & & & $\begin{array}{l}\infty \\
0 \\
0\end{array}$ & $\stackrel{\curvearrowright}{-}$ & & $\stackrel{2}{0}$ \\
\hline$\vec{m}$ & $\dddot{m}$ & Ḧ & & & & & & $\stackrel{m}{0}$ & & $\stackrel{\infty}{\rightarrow}$ & & & & $\stackrel{m}{0}$ & & & $\stackrel{\infty}{-}$ & Iִ & & \\
\hline$\stackrel{\mathcal{N}}{\mathrm{J}}$ & & $\stackrel{\infty}{\infty}$ & $\grave{o}$ & & & & & & & & & $\stackrel{1}{0}$ & 3 & $\stackrel{3}{\circ}$ & & & $\stackrel{\text { nִ }}{\sim}$ & $\stackrel{m}{+}$ & $\stackrel{\overbrace{}}{\rightarrow}$ & $\ddot{3}$ \\
\hline 극 & $\stackrel{?}{0}$ & $\stackrel{n}{i}$ & & $N$ & & & $\stackrel{3}{0}$ & & & $\stackrel{m}{0}$ & & $\stackrel{\llcorner}{\mathrm{i}}$ & & & $\stackrel{3}{0}$ & & $\stackrel{乛}{-}$ & $\stackrel{\infty}{\circ}$ & $\stackrel{10}{0}$ & \\
\hline 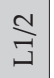 & & $\Lambda$ & $\stackrel{H}{\rightarrow}$ & & $\stackrel{\infty}{\circ}$ & & & $\stackrel{3}{0}$ & & & $\stackrel{\infty}{\circ}$ & - & & $\stackrel{?}{0}$ & & & $\begin{array}{l}\infty \\
\dot{c}\end{array}$ & $\stackrel{+}{-}$ & $\stackrel{\infty}{\infty}$ & \\
\hline$\stackrel{\Xi}{\rightrightarrows}$ & & กี & $\hat{o}$ & $\begin{array}{l}0 \\
\ddot{\infty}\end{array}$ & $\hat{o}$ & & & & تج & ปี & & $\hat{o}$ & & & $\hat{o}$ & $\hat{o}$ & N & $\hat{o}$ & $\stackrel{\llcorner}{O}$ & \\
\hline & 疍 & 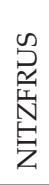 & 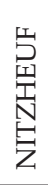 & 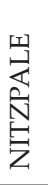 & 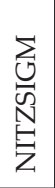 & 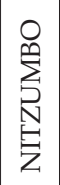 & 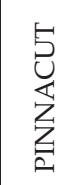 & 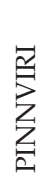 & 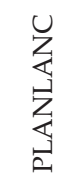 & 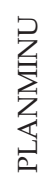 & 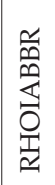 & $\begin{array}{l}0 \\
00 \\
0 \\
0 \\
0 \\
\frac{0}{2}\end{array}$ & 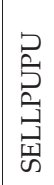 & 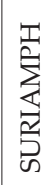 & 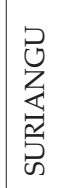 & 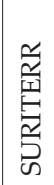 & 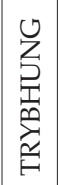 & 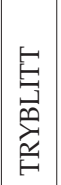 & 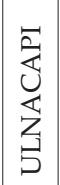 & 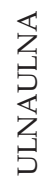 \\
\hline 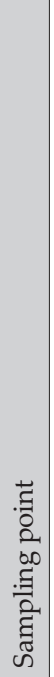 & 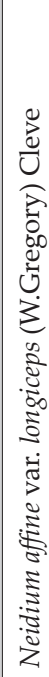 & 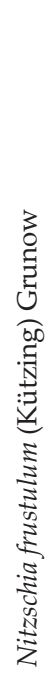 & 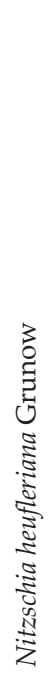 & 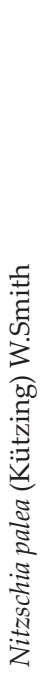 & 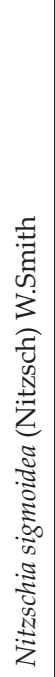 & 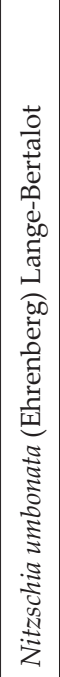 & 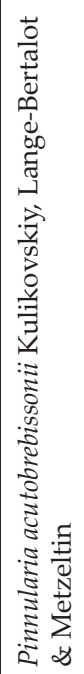 & 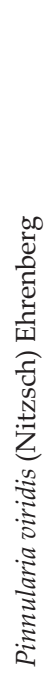 & 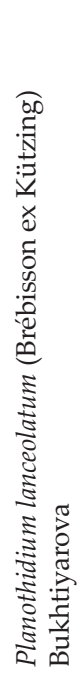 & 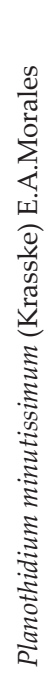 & 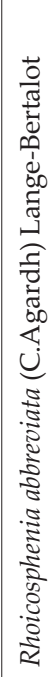 & 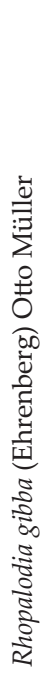 & 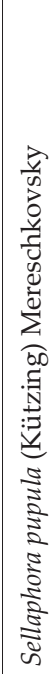 & 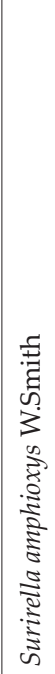 & 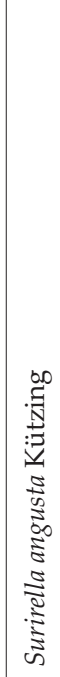 & 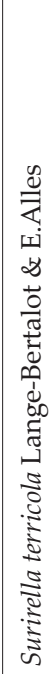 & 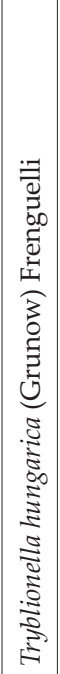 & 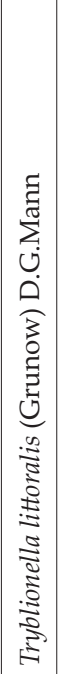 & 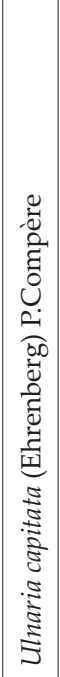 & 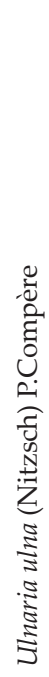 \\
\hline
\end{tabular}


Tab. 4. Values of the Pearson correlation coefficient (r) of the examined parameters and species abundances with first two PCO axes

\begin{tabular}{|c|c|c|}
\hline \multirow[t]{2}{*}{ Parameters and species } & \multicolumn{2}{|c|}{ Pearson correlation Index (r) } \\
\hline & $\begin{array}{c}\text { PCO1 } \\
\text { (38.2\% of total variation) }\end{array}$ & $\begin{array}{c}\mathrm{PCO} 2 \\
\text { (17.6\% of total variation) }\end{array}$ \\
\hline Temp. $\left[{ }^{\circ} \mathrm{C}\right]$ & 0.12 & 0.59 \\
\hline $\mathrm{pH}$ & -0.25 & -0.33 \\
\hline Secchi depth $[\mathrm{cm}]$ & -0.58 & 0.71 \\
\hline Depth [cm] & -0.09 & 0.19 \\
\hline Consumption $\mathrm{KMnO}_{4}$ & -0.02 & 0.04 \\
\hline Floating vegetation [\%] & -0.46 & 0.20 \\
\hline \multicolumn{3}{|l|}{ Species } \\
\hline Amphora pediculus & 0.11 & -0.33 \\
\hline Anomoeoneis sphaerophora & 0.64 & 0.50 \\
\hline Cocconeis placentula var. euglypta & -0.75 & 0.10 \\
\hline Cocconeis placentula var. placentula & -0.07 & 0.23 \\
\hline Cyclotella meneghiniana & -0.53 & 0.41 \\
\hline Fallacia pygmaea & 0.28 & -0.18 \\
\hline Gomphonema pseudoaugur & 0.30 & 0.65 \\
\hline Gomphonema pumilum & -0.32 & 0.63 \\
\hline Gyrosigma acuminatum & -0.47 & -0.48 \\
\hline Hippodonta capitata & 0.13 & -0.38 \\
\hline Lemnicola hungarica & 0.02 & -0.61 \\
\hline Melosira varians & -0.20 & -0.51 \\
\hline Navicula cryptocephala & -0.54 & -0.29 \\
\hline Navicula radiosa & -0.84 & 0.26 \\
\hline Nitzschia frustulum & 0.57 & -0.43 \\
\hline Nitzschia palea & -0.68 & -0.12 \\
\hline Tryblionella hungarica & 0.81 & 0.13 \\
\hline Tryblionella littoralis & -0.04 & 0.33 \\
\hline
\end{tabular}

cient with the second PCO axis and associated lower water temperatures and lower transparency were noted for species Melosira varians, Lemnicola hungarica, Gyrosigma acuminatum, Nitzshia frustulum and Hippodonta capitata (Fig. 4).

Similar abundances in spring and summer were noted for the following species: Cocconeis placentula var. placentula, Fallacia pygmaea, Fragilaria acus (Kützing) Lange-Bertalot, Hippodonta capitata, Lemnicola hungarica, Navicula cryptocephala, Nitzschia palea, Rhopalodia gibba (Ehrenberg) Otto Müller and Tryblionella hungarica. PERMANOVA results did not show significant differences among samples for the season $(p=0.337)$. However, some species showed greater abundance in spring, such as Amphora pediculus Grunow ex A.Schmidt, Cocconeis placentula var. euglypta, Gyrosigma acuminatum, Melosira varians, Navicula radiosa and Sellaphora pupula sensu lato. Other species had higher abundance in summer: Cocconeis pediculus Ehrenberg, Gomphonema pseudoaugur, Nitzschia frustulum, Tryblionella littoralis, Ulnaria capitata (Ehrenberg) P.Compère and Amphora ovalis (Kützing) Kützing 

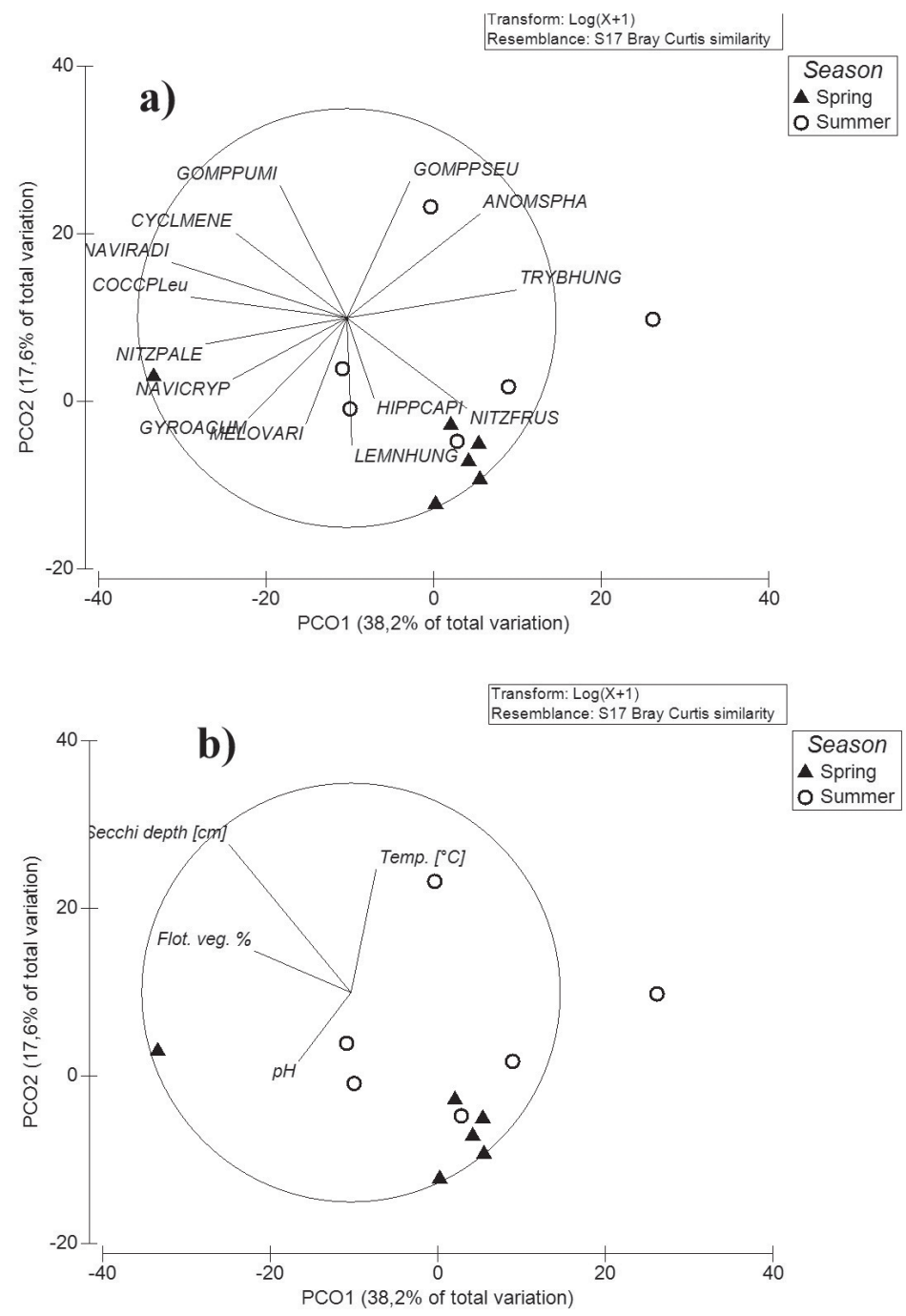

Fig. 4. Principal coordinates analysis (PCO) comparing the community composition between localities in spring and summer season. PCO plot coded by the species vectors indicates taxa positively correlated $(r>0.4)$ with the axes $(a)$ and environmental vectors $(b)$.

\section{DISCUSSION}

The most representative taxa in Šerićka bara marsh (Cocconeis placentula, Gyrosigma acuminatum, Navicula cryptocephala, Nitzschia palea and Cyclotella meneghiniana) were also described in similar investigations of epipelic communities in 16 and 45 fishponds in the Czech Republic (Lysáková et al., 2007; PoulíčKová et al., 2009, respectively). However, species that were abundant in these fishponds, such as Gomphonema parvulum (Kützing) Kützing, Nitzschia acicularis (Kützing) W.Smith, Tabellaria flocculosa (Roth) Kützing and Aulacoseira spp., were not recorded in this study. Unlike the fishponds, the species Fallacia 
pygmaea was represented in this study in almost every sample with abundance of up to $6.3 \%$. According to Hoffman et al. (2011), this species is common in brackish water or freshwater rich in electrolytes. Lemnicola hungarica, a common epiphyte on the roots of Lemnaceae species such as Lemna minor, L. gibba and Wolffia arrhiza (BuczKó, 2007) in slowflowing channels and lowland marshes with high trophic status and medium high to high electrolyte contents was also relatively abundant. In Śerićka bara marsh it was very abundant in epipelon, since the floating vegetation of Lemnaceae species covering the marsh could provide a habitat from which L. hungarica could easily detach and fall to the bottom. However, no positive correlation between coverage of floating vegetation and abundance of L. hungarica was found, probably due to wind impact on this floating cover. Anomoeoneis sphaerophora, a typically brackish species which is common in lowland waters with high electrolyte content, was also common in this study (Hofmann et al., 2011).

Epipelic algae have been used as bioindicators in deep-water ecosystems (SMOL \& STOERMER, 2010). However, PoulíčKová et al. (2004) speculated that littoral sediments contain a mixture of planktonic, epiphytic and epipelic algae which are rarely taken into account. They have established significant differences among epilithic, epiphytic and epipelic samples in evaluation of the trophic states of peri-alpine lakes in Austria, where epipelon overestimated the trophic status when compared to the trophic status deduced from open water samples. HAšLER et al. (2008) reported that epipelic assemblages reflect sediment quality rather than water-column chemistry. However, a study by KITNER \& PoulíčKová (2003) showed that no significant differences in index values for epipelic, epiphytic and epilithic communities in littoral part of the shallow lakes exist. Considering that diatom indices can give different results in the evaluation of trophic status, two diatom indices were used in this study. Both of them indicated ongoing eutrophication processes in the marsh, followed by the massive growth of emergent and floating vegetation, due which the area has a tendency to become a terrestrial ecosystem. The greater presence of dominant epipelic species in this study also indicated high trophic status and electrolyte-rich water. Species Hippodonta capitata, Tryblionella hungarica, Navicula cryptocephala and Gyrosigma acuminatum are common in eutrophic and polytrophic waters of $\beta$ - to $\alpha$-mesosaprobic zone, while very abundant species Craticula cuspidata is also known in industrial waste waters (Hofmann et al., 2011). Following the classification of van DAm et al. (1994) most frequent and dominant taxa in the Šerićka bara marsh were alkaliphilous and alkalibiontic, fres brakish, nitrogen autotrophic or facultatively nitrogen-heterotrophic taxa, needing periodically elevated concentrations of organically bound nitrogen, with moderate oxygen requirements, indicating eutrophic state. Moreover, the marsh is surrounded by agricultural land and villages that greatly affect the eutrophication processes in it.

Using diatoms known for cryptic and pseudocryptic diversity, such as Navicula cryptocephala and Sellaphora pupula sensu lato, in bioindication of water quality becomes difficult. Finlay et al. (2002) discussed Cyclotella meneginiana, Nitzschia palea and Navicula cryptocephala as widely distributed and ecologically eurivalent species, but detection of cryptic and pseudocryptic diversity introduced doubts concerning their cosmopolitan distribution (Poulíč́ová et al., 2008; PoulíčKová et al., 2010). Species Navicula cryptocephala was the most common species in epipelic community of the Šerićka bara marsh. Since this study gives only the first results on epipelic communities, more detailed studies about taxonomy and distribution of heteregoneous species in the geographic area of Bosnia and Herzegovina are needed.

Light and temperature significantly influence diatom abundance in different communities (Hill, 1996). Poulíč́ová et al. (2014) stressed that epipelon distribution is affected 
primarily by the depth of the overlying water, light, temperature, and sediment type. The most influential factor in this investigation was water transparency, which is in accordance with Goldenberg et al. (2014), who also pointed out the influence of particles that decrease transparency, and thus change the structure of diatom communities. The increased abundance of facultatively heterotrophic species Cyclotella meneghiniana (LyLIS \& TrAINOR, 1973) in epipelic communities is probably the consequence of decreased transparency and limitation by light in lower water layers. PouličKova et al. (2014) showed that diatom epipelic communities are closely related to environmental gradients, $\mathrm{pH}$ and conductivity. However, except for the coverage of floating vegetation, the ecological parameters in this study were in quite narrow ranges, most likely too narrow to influence shifts in diatom assemblages in the research period, thus making it difficult to distinguish the most relevant parameters driving the structure and composition of epipelic diatom communities.

Seasonally, the greatest differences among the investigated sites were noted for lower water temperature ranges, accompanied by increased abundance of Gomphonema acuminatum and Cymatopleura solea in spring, in relation to summer, when increased abundance of Amphora ovalis was noted. Seasonal variations in epipelic communities were observed by ŠPAčKOvA et al. (2009), with a greater presence of cynobacteria in summer due to increase of organic matter content. As in this study, Sellaphora pupula sensu lato was the most abundant in spring months, and Navicula cryptocephala, Achnanthidium minutissiumum and Nitzschia palea were more or less equally distributed during both seasons. The species Sellaphora pupula sensu lato was observed with an occurrence of up to $1.5 \%$, which was in accordance with the results of ŠPAčKOvA et al. (2009), whilst PouličKova et al. (2008) found this species in up to $40 \%$ of epipelon samples of British lakes. Since this species has been found only in spring, it is possible that higher distribution of Sellaphora pupula sensu lato in British waters is influenced by lower water temperature and different climate conditions.Although there were variations in the abundances of several species during spring and summer, diversity index values remained quite constant throughout both the research seasons.

\section{CONCLUSION}

Epipelic communities of the Šerićka bara marsh presented a high diversity of diatoms, and most of identified species were eutraphentic and alkaliphilous, indicating the high eutrophic status of the marsh. No statistical difference in diatom communities between spring and summer was established, although several species had more or less different abundances during these two sampling periods. The eutrophication process is followed by the dense growth of floating and emerged vegetation. Considering the growing worldwide threat to marshes, measures for conservation of this marsh, currently a completely unprotected ecosystem, are desperately needed in order to secure the habitat for many marsh species.

Received April 28, 2016

\section{REFERENCES}

Anderson, M.J., Gorley, R.N. \& Clarke, K.R., 2008: PERMANOVA+ for PRIMER: Guide to Software and Statistical Methods. PRIMER-E, Plymouth, United Kingdom.

BuczKó, K., 2007: The occurrence of the epiphytic diatom Lemnicola hungarica on different European Lemnaceae species. Fottea 7(1), $77-84$. 
Clarke, K.R. \& Gorley, R.N., 2006: PRIMER v6: User Manual/Tutorial. PRIMER-E, Plymouth, United Kingdom.

DodDs, W.K., 2003: The role of periphyton in phosphorus retention in shallow freshwater aquatic systems. Journal of Phycology 39, 830 - 849 .

Goldenberg Vilar, A., Sileno, G., Alvarez, S., van Dam, H., van Loon, E.E., Vonk, J.A., van Der Geest, H.G. \& AdmiraAl, W., 2014: Typology of diatom communities in the Dutch delta: Recognizing patterns of environmental drivers in nutrient rich ditches. Ecological indicators 45, 561- 569.

Gómez, N., 1998: Use of epipelic diatoms for evaluation of water quality in the Matanza Riachuelo (Argentina), a pampean plain river. Water Research 32, 2029 - 2034.

Guiry, M.D. \& Guiry, G.M., 2015: AlgaeBase. World-wide electronic publication, National University of Ireland, Galway. http://www.algaebase.org [accessed on February 1, 2015].

Hafner, D., Jasprica, N. \& CArić, M., 2013: Epiphytic diatoms on Nymphaea alba L. leaves in a Submediterranean wetland (South Bosnia and Herzegovina). Natura Croatica 22(2), 319 - 331.

Hašler, P., Štěpánková, J., Špačková, J., Neustupa, J., Kitner, M., Hekera, P., Veselá, J., Burian. J. \& PoulíčKovÁ, A. 2008: Epipelic cyanobacteria and algae: a case study from Czech ponds. Fottea 8(2), 133-146.

Hill, W., 1996: Effects of light. In: Stevenson, R. J., Bothwell, M. L. \& Lowe, R. L. (eds) Algal Ecology: Freshwater Benthic Ecosystems. Academic Press, San Diego, 57 - 76.

Hofmann, G., Werum, M. \& Lange-Bertalot, H., 2011: Diatomeen im Süßwasser - Benthos von Mitteleuropa. Bestimmungsflora Kieselalgen für die ökologische Praxis. Über 700 der häufigsten Arten und ihre Ökologie. A.R.G. Gantner Verlag K.G., Rugell, pp. 908

Hustedt, F., 1930: Bacillariophyta. In: Pascher, A. (ed.): Die Süsswasserflora Mitteleuropas 10. Jena.

Kitner, M. \& PoulíčKová, A., 2003: Littoral diatoms as indicators for the eutrophication of shallow lakes. Hydrobiologia 506, $519-524$.

Krammer, K. \& Lange - Bertalot, H., 1986: Bacillariophyceae: Naviculaceae. In: Ettr, H., Gerloff, J., Heynig, H. \& Mollenhauer, D. (eds), Süßwasserflora von Mitteleuropa, vol. 2/1, pp. 1-876. Gustav Fischer Verlag, Sttutgart.

Krammer, K. \& Lange - Bertalot, H., 1988: Bacillariophyceae: Bacillariaceae, Epithemiaceae, Surirellaceae. In: Ettr, H., Gerloff, J., Heynig, H. \& Mollenhauer, D. (eds), Süßwasserflora von Mitteleuropa, vol. 2/2, pp. 1-596. Gustav Fischer Verlag, Sttutgart.

Krammer, K. \& Lange - Bertalot, H., 1991a: Bacillariophyceae: Centrales, Fragilariaceae, Eunotiaceae. In: Ettl, H., Gerloff, J., Heynig, H. \& Mollenhauer, D. (eds), Süßwasserflora von Mitteleuropa, vol. 2/3, pp. 1-576. Gustav Fischer Verlag, Sttutgart.

Krammer, K. \& Lange - Bertalot, H., 1991b: Bacillariophyceae: Achnanthaceae, Kritische Ergänzungen zu Navicula (Lineolatae) und Gomphonema. Gesamtliteraturverzeichnis. In: Ettr, H., Gerloff, J., HeyNIG, H. \& Mollenhauer, D. (eds), Süßwasserflora von Mitteleuropa, vol. 2/4, pp. 1-437. Gustav Fischer Verlag, Sttutgart.

Krammer, K., 2000: The genus Pinnularia. Diatoms of Europe, Volume 1. A.R.G. Gantner Verlag K.G. Florida. 703 pp.

Krammer, K., 2002. Cymbella. Diatoms of Europe, Volume 3. A.R.G. Gantner Verlag K.G. Florida. 584 pp.

Krammer, K., 2001. Navicula sensu stricto 10 Genera Separated from Navicula sensu lato Frustulia. Diatoms of Europe, Volume 2. A.R.G. Gantner Verlag K.G. Florida. 526 pp.

Kulijer, D. \& Šımı́́, E., 2011: Preliminarna studija „lstraživanje biološke raznolikosti i eko-turističkih potencijala Šerićke bare i jezera Modrac". Ornitološko društvo "Naše ptice". Sarajevo.

Lolić, S., Matavulj, M. \& Dekić, R., 2015: Distribution of phytoplankton at fish pond prevlaka (Bardača). Zbornik predavanja. 7. International Conference „Water \& Fish” Faculty of Agriculture, BelgradeZemun, Serbia.

Lylis, J. C. \& Trainor, F. R., 1973: The heterotrophic capabilities of Cyclotella meneghiniana. Journal of Phycology 9 (4), $365-369$.

Lysáková, M., Kitner, M. \& PoulíčKová, A., 2007: The epipelic algae of fishponds of Central and Northern Moravia (The Czech Republic). Fottea 7 (1), 69 -75.

Moulten, T.P., De Souza, M.L., Silveira, R.M.L. \& Krsulovic, F.E.M., 2004: Effects of ephemeropterans and shrimps on periphyton and sediments in a coastal stream (Atlantic forest, Rio De Janeiro, Brazil). Journal of the North American Benthological Society 23, 868-881.

Poulíč́ová, A., HašLer, P., Lysáková, M. \& Spears, B., 2008: The ecology of freshwater epipelic algae: an update. Phycologia, 47 (5), $437-450$. 
PoulíčKová, A., Duchoslav, M. \& Dokulil, M., 2004: Littoral diatom assemblages as bioindicators of lake trophic status: A case study from perialpine lakes in Austria. European Journal of Phycology. 39, 143-152.

PoulíčKová, A., Dvǒ̌Ák, P., Mazalová, P. \& HašLer, P., 2014: Epipelic microphototrophs: an overlooked assemblage in lake ecosystems. Freshwater Science 33 (2), 513 - 523.

PoulíčKová, A., Neustupa, J., ŠPaČKová, J. \& ŠKaloud, P., 2009: Distribution of epipelic diatoms in artificial fishponds along environmental and spatial gradients. Hydrobiologia. 624 (1), 81-90.

Poulíčková, A., ŠpačKová, J., Kelly, M.G., Duchoslav, M. \& Mann D.G., 2008: Ecological variation within Sellaphora species complexes (Bacillariophyceae) - specialists or generalists? Hydrobiologia 614, 373-386.

PoulíčKovÁ, A., Veselá, J., Neustupa, J. \& ŠKAloud, P., 2010: Pseudocryptic diversity versus cosmopolitanism in diatoms: a case study on Navicula cryptocephala Kütz. (Bacillariophyceae) and morphologically similar taxa. Protist 161, $353-369$.

Redžıć, S., Barudanović, S. \& Radević, M. (ed.), 2008: Bosna i Hercegovina - Zemlja raznolikosti, Pregled i stanje biološke i pejzažne raznolikosti Bosne i Hercegovine, Prvi izvještaj BiH za CBD, Bemust, Sarajevo.

Rott, E., Pfister, P., van Dam, H., Pipp, E., PAll, K., Binder, N. \& Ortler, K., 1999: Indikationslisten für Aufwuchsalgen in Fließgewässern Österreichs, Teil 2: Trophieindikation und autökologische Anmerkungen. Wasserwirtschaftskataster. - Bundesministerium für Land- und Forstwirtschaft. Wien.

Round, F.E., 1953: An investigation of two benthic algal communities in Malham Tarn, Yorkshire. Journal of Ecology 41, $174-179$.

Shannon, C. E. \& Wiener, W., 1949: The Mathematical theory of Comunication. University of Illionis Press, Urbana, 125.

Smol, J.P. \& Stoermer, E.F., 2010: The Diatoms: Applications for Environmental and Earth Sciences. Second Edition, Cambridge University Press. Cambridge, UK.

ŠPAČKová, J., HAŠLER, P., ŠTĚPÁNKová, J.\& PoulíčKová, P., 2009: Seasonal succession of epipelic algae: a case study on a mesotrophic pond in a temperate climate. Fottea 9 (1), 121-133.

Trajković, J., Baras, J., Mirić, M. \& Šiler, S., 1983: Analiza životnih namirnica, Tehnološko-metalurški fakultet, Beograd.

van Dam, H., Mertens, A., Sinkeldam, J., 1994: A coded checklist and ecological indicator values of freshwater diatoms from the Netherlands. Netherlands Journal of Aquatic Ecology 28, 117 - 133.

Velasco, J., Millan, A., Vidal-Abarca, M.R., Suarez, M.L., Guerrero, C. \& Ortega, M., 2003: Macrophytic, epipelic, and epilithic primary production in a semiarid Mediterranean stream. Freshwater Biology 48, 1408 - 1420.

YANG, J.R. \& Dickman, M., 1993: Diatoms as indicators of lake trophic status in Central Ontario, Canada. Diatom Res. 8, 179 - 193.

Google EArth, 2010/Tele Atlas 2010/Image 2010 Digital Globe, World-wide electronic publication.

\title{
SAŽETAK
}

\section{Epipelonske dijatomeje Šerićke bare (sjeveroistočna Bosna i Hercegovina)}

\author{
J. Kamberović, K. Kralj Borojević, A. Razić \& S. Barudanović
}

Močvare su jedan od najugroženijih ekosustava u Bosni i Hercegovini, ograničenih na male površine zbog učestalih zahvata melioracije i isušivanja. Iako do sada nisu provedena istraživanja zajednica primarnih proizvođača na području Šerićke bare u sjeveroistočnoj Bosni i Hercegovini, ona je prepoznata kao značajno močvarno stanište u nacionalnim programima ocjene biološke raznolikosti. U radu su istraživane epipelonske dijatomeje, značajni pokazatelji promjena u vodenim ekosustavima. Ciljevi istraživanja bili su: 1) uvrditi dijatomejski sastav bentoskih zajednica Šerićke bare, 2) uporediti strukturu dijatomeja tijekom proljetnog i ljetnog razdoblja te 3) procijeniti trofički status ove močvare. Utvrđeno je 57 
vrsta dijatomeja iz 33 roda. Najveći broj vrsta pripadao je rodovima Gomphonema (8) i Nitzschia (5). Najbrojnije vrste bile su Melosira varians, Gyrosigma acuminatum, Lemnicola hungarica, Navicula cryptocephala, Cocconeis placentula var. placentula, Hippodonta capitata, Fallacia pygmaea, Anomoeoneis sphaerophora, Craticula cuspidata, Cyclotella meneghiniana i Tryblionella hungarica. Shannon-Wienerov indeks raznolikosti varirao je od 2,47 do 2,96 ukazujući na visoku raznolikost vrsta u epipelonskim zajednicama. Trofički indeks prema Rott-u kretao se u vrijednostima od 2,7 do 3,4 i ukazujući na eupolitrofni do politrofni status močvare. Iako analizom PERMANOVA nisu utvrđene statistički značajne razlike u strukturi zajednice proljetnih i ljetnih uzoraka epipelona, nekoliko je vrsta imalo veću brojnost u proljeće, dok su druge bile zastupljenije u ljeto. Povećanjem temperature povećala se brojnost vrsta Amphora ovalis i Caloneis sp., dok su u proljetnoj sezoni s nižim temperaturama vode bile zastupljene Gomphonema angustum, Cocconeis pediculus, Cymatopleura solea, Gomphonema acuminatum i Gyrosigma acuminatum. Dominatne vrste $\mathrm{u}$ epipelonu istraživane močvare ukazuju na visoki stupanj trofije i vodu bogatu elektrolitima. Procese eutrofikacije prati bujni porast emerzne i flotantne vegetacije. Imajući u vidu sve veću ugroženost močvara u svijetu, mjere očuvanja ovog ekosustava, koji trenutno nažalost ne podliježe nikakvoj zaštiti, neophodne su zbog osiguranja staništa za mnoge biljne i životinjske močvarne vrste. 